\title{
Increasing feasibility and patient comfort of MRI in children with juvenile idiopathic arthritis
}

\author{
Robert Hemke • Mira van Veenendaal • \\ Taco W. Kuijpers • Marion A. J. van Rossum • \\ Mario Maas
}

Received: 20 May 2011 /Revised: 30 August 2011 / Accepted: 17 September 2011 /Published online: 13 January 2012

(C) The Author(s) 2011. This article is published with open access at Springerlink.com

\begin{abstract}
Background MRI is the most sensitive imaging modality in juvenile idiopathic arthritis (JIA), but has practical limitations. Optimizing the scanning protocol is, therefore, necessary to increase feasibility and patient comfort.

Objective To determine the feasibility of bilateral noncontrast-enhanced open-bore MRI of knees and to assess the presence of literature-based MRI features in unsedated children with JIA.

Materials and methods Children were classified into two clinical subgroups: active arthritis (group $1 ; n=29$ ) and inactive disease (group $2 ; n=18$ ). MRI features were evaluated using a literature-based score, comprising synovial hypertrophy, cartilage lesions, bone erosions, bone marrow changes, infrapatellar fat pad heterogeneity, effusion, tendinopathy and popliteal lymphadenopathy.

Results The MRI examination was successfully completed in all 47 children. No scan was excluded due to poor image quality. Synovial hypertrophy was more frequent in group 1
\end{abstract}

R. Hemke $(\bowtie) \cdot$ M. Maas

Department of Radiology, Academic Medical Center,

Meibergdreef 9 ,

1105AZ Amsterdam, The Netherlands

e-mail: r.hemke@amc.nl

R. Hemke $\cdot$ M. van Veenendaal $\cdot$ T. W. Kuijpers

M. A. J. van Rossum

Department of Pediatric Hematology, Immunology, Rheumatology and Infectious Disease, Emma Children's Hospital AMC,

Meibergdreef 9 ,

1105AZ Amsterdam, The Netherlands

M. A. J. van Rossum

Department of Pediatric Rheumatology, Jan van Breemen Institute,

Dr. Jan van Breemenstraat 2,

Amsterdam, The Netherlands
(36.2\%), but was also seen in $19.4 \%$ of the knees in group 2 . Infrapatellar fat pad heterogeneity was more prevalent in group $2(86.1 \% ; P=0.008)$. Reproducibility of the score was good (Cohen kappa, 0.49-0.96).

Conclusion Bilateral non-contrast-enhanced open-bore knee MRI is feasible in the assessment of disease activity in unsedated children with JIA. Signs differing among chidren with active and inactive disease include infrapatellar fat pad heterogeneity and synovial hypertrophy.

Keywords Magnetic resonance imaging · Juvenile idiopathic arthritis $\cdot$ Knee joint

\section{Introduction}

Juvenile idiopathic arthritis (JIA) is not a solitary entity, but a term that encompasses all forms of arthritis that begin before the age of 16 years, persist for more than 6 weeks, and are of unknown etiology and pathophysiology [1]. Studies in developed countries have reported a prevalence that varies between 16 and 150 per 100,000. Therefore, it is one of the most common chronic diseases in childhood and represents one of the leading causes of acquired disability in children [1]. It is characterized by inflammation of hypertrophic synovium that can lead to pain, loss of function and destruction of joints [1].

Within the past 10 years, the use of MRI and advances in MRI techniques have significantly improved evaluation of joint pathologies in adults with rheumatoid arthritis [2]. Despite the large number of studies available in adults, experience on the use of MRI in the assessment of JIA is limited. Despite technical limitations MRI is deemed the preferred imaging modality for detecting synovial inflammation and early erosive changes in JIA [3]. Limitations 
include the necessity for sedation in young children, the risk of an allergic reaction when intravenous contrast is administered and the limited number of joints that can be evaluated during one imaging session because of time constraints [3]. As these practical limitations potentially hamper the benefits of MRI in the assessment of disease activity, optimizing the scanning protocol is necessary to increase patient comfort and feasibility. Further, knowledge of the normal developmental process of the knee joint in children is needed, since some MRI findings are part of maturation and should not be mistaken for pathologies [4].

Using an intravenous contrast agent prolongs the examination time, increases invasiveness and patient discomfort, and thereby reduces the feasibility of MRI. Therefore, the disadvantages of omitting intravenous contrast may be outweighed by the possibility to assess more joints and by greater feasibility [5]. Another limitation is the spatial confinement of cylindrical MRI systems, which might contribute to increased anxiety in children [6]. An open-bore MRI system might alleviate this limitation. The open system allows a parent close contact with a child and allows the child contact with the outside world. This further reduces invasiveness and patient discomfort.

Many uncertainties remain for the diagnostic accuracy and implementation of MRI to assess peripheral joints in JIA in a standardized manner [3]. One challenge is the limited experience in evaluating MRI findings at different stages of disease activity in JIA. Therefore, the aim of our study was to determine the feasibility of non-sedated bilateral non-contrast enhanced open-bore MRI and to assess the presence of established MRI features in unsedated children with JIA and current or previous knee involvement at different stages of disease activity.

\section{Materials and methods}

\section{Patients}

Data of all consecutive children with MRI evaluations of the knees between November 2007 and October 2008 were included in this prospective observational study. Indications for MRI evaluation were clinical active arthritis suspicious for JIA (group 1), or follow-up of children with JIA and clinically inactive disease who had a history of clinically evident arthritis in at least one knee (group 2). Children visited one of the outpatient clinics of two tertiary pediatric rheumatology centers (Emma Children's Hospital/Academic Medical Center and Jan van Breemen Institute, both in Amsterdam, The Netherlands). At the time of presentation, all children had clinical and laboratory assessments followed by a radiological evaluation of both knees consisting of conventional radiographs and MRI. Children who had had an intra-articular corticosteroid injection in the last 6 months were excluded. The study was performed in accordance with the Declaration of Helsinki and the local medical ethical regulations. Consultation with our institutional review board revealed that informed consent was not necessary, since the MRI approach described is noninvasive.

Children in group 1 met the criteria for JIA defined as clinical arthritis in $\geq 1$ joint for $>6$ weeks, suggestive for JIA. After a period of 6 months, all newly diagnosed children with JIA were clinically re-evaluated, and reclassified if necessary [7]. In group 2, clinically inactive disease was defined according to the preliminary criteria for inactive disease in JIA by Wallace et al. [8].

\section{Clinical assessment}

Clinical assessment included a 67-joint count defining the presence of swelling, pain on motion/tenderness and limited range of motion. A physician's global assessment of overall disease activity, a patient's global assessment of overall wellbeing and an assessment of patient's pain were all measured on a $100-\mathrm{mm}$ visual-analogue scale. Functional ability was evaluated using the Dutch version of the childhood health assessment questionnaire (CHAQ) [9, 10]. Laboratory tests included the erythrocyte sedimentation rate (ESR) and the C-reactive protein (CRP) level.

\section{MRI protocol}

To optimize the feasibility of the MRI examination, intravenous contrast injection was omitted and an open-bore MRI scanner was used. Therefore, non-contrast-enhanced MRI of both knees was performed using an open-bore 1-T MRI system (Panorama HFO; Philips Medical Systems, Best, The Netherlands) with a dedicated knee coil. No sedation was used. MRI sequences included sagittal T2-weighted fatsaturated images (TR, 4,000-6,000 ms; TE, $30 \mathrm{~ms}$; slice thickness, $4 \mathrm{~mm}$; field of view, $150 \times 92 \mathrm{~mm}$; matrix, $232 \times$ 186; bandwidth, $218.6 \mathrm{~Hz} /$ pixel), coronal T2-weighted fatsaturated images (TR, 2,700-4,500 ms; TE, $50 \mathrm{~ms}$; slice thickness, $4 \mathrm{~mm}$; field of view, $150 \times 114 \mathrm{~mm}$; matrix, $248 \times 195$; bandwidth $169.6 \mathrm{~Hz} /$ pixel), axial T2-weighted fat-saturated images (TR, 2,900-4,300 ms; TE, $50 \mathrm{~ms}$; slice thickness, $4 \mathrm{~mm}$; field of view, $150 \times 114 \mathrm{~mm}$; matrix, $248 \times$ 195; bandwidth, $169.6 \mathrm{~Hz} /$ pixel), sagittal T1-weighted images (TR, 450-650 ms; TE, $10 \mathrm{~ms}$; slice thickness, $4 \mathrm{~mm}$; field of view, $150 \times 92 \mathrm{~mm}$; matrix, $221 \times 234$; bandwidth, $178.7 \mathrm{~Hz} /$ pixel), and axial turbo spin-echo proton density-weighted images (TR, 3,000-5,000 ms; TE, $30 \mathrm{~ms}$; slice thickness, $4 \mathrm{~mm}$; field of view, $170 \times 124 \mathrm{~mm}$; matrix, $340 \times 252$; bandwidth, $246.3 \mathrm{~Hz} /$ pixel). The total scan time was $41 \min 28 \mathrm{~s}$ for both knees. 
Image analysis

MR images were interpreted in consensus by one expert musculoskeletal radiologist (MM; 16 years of experience in musculoskeletal radiology) and a radiology trainee (RH; 3 years of experience in musculoskeletal radiology) masked to clinical history, the duration, extent and severity of the symptoms. A newly composed literaturebased grading score was used for assessment [2, 11-16]. Each knee was assessed at the time of MRI interpretation according to the following criteria:

1. Synovial hypertrophy. The synovium was assessed for signal intensity characteristics and maximal thickness and was evaluated at six sites of the knee joint; patellofemoral, suprapatellar recesses, infrapatellar fat pad, adjacent to the anterior and posterior cruciate ligaments, adjacent to the medial posterior condyle and lateral posterior condyle. Increased synovial thickness of $\geq 3 \mathrm{~mm}$ was considered definitely abnormal [11]. Synovial thickness was scored based on the maximum thickness in any section as follows: grade 0 if $<3 \mathrm{~mm}$, grade 1 if $\geq 3 \mathrm{~mm}$.

2. Articular cartilage lesions. As progressive JIA may lead to cartilage destruction, the articular cartilage was assessed for focal destruction (intact, superficial loss and/or thinning, or deep erosions to subchondral bone) [11]. The cartilage was scored for the presence of lesions at the patella, at the medial and lateral femur, and at the medial and lateral tibia plateau. Cartilage lesions were scored as absent (grade 0) or present (grade 1).

3. Bone erosions. Bone erosions were defined according to the OMERACT RAMRIS guidelines as sharply marginated bone lesions with low signal intensity in T1weighted images in at least two planes, with a cortical break present in at least one imaging plane [2]. The bone was scored for the presence of erosions in the patella, in the medial and lateral femur, and in the medial and lateral tibia plateau (scored as grade 0 if not present, grade 1 if present).

4. Bone marrow changes, suggestive for bone marrow edema. Bone marrow changes, suggestive for bone marrow edema, were defined as lesions within the trabecular bone, with ill-defined margins and high signal intensity on T2-weighted fat-saturated and low signal intensity on T1-weighted images. The bone marrow was scored for the presence of changes in the patella, in the medial and lateral femur, and in the medial and lateral tibia plateau (scored as grade 0 if not present, grade 1 if present).

5. Heterogeneity of the infrapatellar fat pad. Infrapatellar fat pad heterogeneity correlates with synovial thickness in early JIA [11] and was evaluated (scored as: 0, absent; 1, minimal; 2, marked). In addition, fat pad heterogeneity was further classified as being caused by water infiltration or by scar tissue. On MRI, water infiltration of the infrapatellar fat pad is depicted as areas of decreased signal intensity on T1-weighted images and increased signal intensity on $\mathrm{T} 2$-weighted fat sat images, whereas infiltration of the infrapatellar fat pad by scar tissue shows decreased signal intensity on both T1-weighted and T2-weighted images [12].

6. Joint effusion. The extent of effusion was subjectively graded within the suprapatellar recesses, the lateral recesses, Baker cyst and central recesses (scored as: 0, absent; 1, minimal; 2, marked) [11, 13-15].

7. Tendinopathy/internal derangement. The medial collateral ligament, lateral collateral ligament, anterior cruciate ligament, posterior cruciate ligament, patellar tendon and the menisci were assessed for pathology (scored as: 0, normal; or 1, abnormal).

8) Popliteal lymph nodes. The number of popliteal lymph nodes has been reported to correlate with the amount of inflamed synovial volume in rheumatoid arthritis (RA) patients and their presence has also been described in JIA $[11,14,16]$. Therefore, the number of popliteal lymph nodes was determined.

The primary analyses were based on the first reading. To get an impression of reproducibility, the same readers reviewed the complete data set of MR images 2 years later in an identical manner. The second scoring took place without knowledge of the results of the first.

All children had conventional radiographs taken of both knees within 2 weeks of the MRI examination. For the purpose of this study, the knee radiographs were reviewed and scored in consensus by an experienced pediatric rheumatologist (MvR; 10 years of experience in musculoskeletal radiology) and a radiology trainee (RH; 3 years of experience in musculoskeletal radiology), according to the Dijkstra composite score [17]. The readers were masked to the clinical status and MRI findings.

\section{Statistical analysis}

Descriptive statistics were reported in terms of medians and interquartile ranges for the continuous variables and in terms of absolute frequencies and percentages for the categorical variables. The Mann-Whitney $U$ test, Fisher exact test, and the chi-square test were used to analyze differences between study groups. All tests assumed a two-tailed probability. A $P$ value of less than 0.05 indicated a significant difference. The Cohen kappa coefficient was used to quantify the agreement between observer scores [18]; agreement was 
classified as follows: $\kappa<0.40=$ poor, $\geq 0.40-0.60=$ moderate, $>0.60-0.80=$ substantial and $>0.80=$ good agreement. SPSS Statistics (version 16.0; SPSS, Chicago, IL) was used for all analyses. Statistical analyses were performed in close collaboration with a clinical epidemiologist.

\section{Results}

Patient characteristics

Data of 51 children were collected. Four were excluded: one diagnosed by MRI with a rupture of the anterior cruciate ligament, and three who initially were diagnosed with JIA but were later reclassified because of a non-rheumatological ailment. Therefore, the data comprised 94 sets of MR images from 47 children with JIA. Table 1 shows the clinical characteristics of the children. Age (Mann-Whitney $U$ test, $P=0.299$ ) and gender (Fisher exact test, $P=0.493$ ) were comparable between the two study groups. Overall disease activity (chi-square test), joint and CHAQ scores (MannWhitney $U$ test) were significantly lower in group 2 compared with group 1 .

\section{Imaging findings}

The complete MRI examination of both knees was successfully completed by all 47 children, the youngest being 5 years of age. No MRI dataset was excluded due to poor image quality. The presence of the scored MRI features for the respective study groups is depicted in Fig. 1.

An extensive overview of MRI findings in the studied groups is presented in Table 2. Synovial hypertrophy (Fig. 2) tended to be present more frequently in group 1, but the difference did not reach statistical significance $(36.2 \%$ vs $19.4 \%$ respectively, Fisher exact test $P=0.106)$. Synovial hypertrophy was detected in seven out of 36 (19.4\%) knees in six out of $18(33 \%)$ children in group 2 (Table 2$)$. These

Table 1 Patient characteristics at study entry of 47 children with juvenile idiopathic arthritis (JIA) ${ }^{\mathrm{a}}$

\begin{tabular}{|c|c|c|c|}
\hline & All patients $n=47$ & Group 1 (active JIA) $n=29$ & Group 2 (inactive JIA) $n=18$ \\
\hline Male, $n(\%)$ & $12(25.5)$ & $6(20.7)$ & $6(33.3)$ \\
\hline Female, $n(\%)$ & $35(74.5)$ & $23(79.3)$ & $12(66.7)$ \\
\hline Age at inclusion, years & $11.6(9.2-14.1)$ & $12.1(9.2-14.6)$ & $11.0(9.6-13.5)$ \\
\hline Disease duration at inclusion, years & $1.8(0.3-4.0)$ & $0.4(0.1-2.6)$ & $3.7(1.9-8.1) * * *$ \\
\hline Inactive disease, years & - & - & $0.8(0.5-0.9)$ \\
\hline $\begin{array}{l}\text { Physician's global assessment of } \\
\text { overall disease activity, } \mathrm{mm}^{\mathrm{b}}\end{array}$ & $14(1-37)$ & $31(18-47)$ & $0(0-4) * * *$ \\
\hline $\begin{array}{l}\text { Patients' global assessment of } \\
\text { overall well-being, } \mathrm{mm}^{\mathrm{b}}\end{array}$ & $17(1-52)$ & $28(14-54)$ & $4(0-21) *$ \\
\hline Patients' pain assessment, $\mathrm{mm}^{\mathrm{b}}$ & $24(1-52)$ & $46(10-62)$ & $7(0-25) *$ \\
\hline C-HAQ score, units ${ }^{c}$ & $0.375(0.125-1.375)$ & $0.625(0.250-1.375)$ & $0.125(0-0.250) * *$ \\
\hline Number of actively inflammed joints & $1(0-3)$ & $2(1-4)$ & $0(0-0) * * *$ \\
\hline $\begin{array}{l}\text { Number of joints with limited range-of-motion } \\
\text { Overall disease activity }{ }^{\mathrm{d}}, n(\%)\end{array}$ & $1(0-2)$ & $2(1-4)$ & $0(0-2) * * *$ \\
\hline - Inactive & $18(38.3)$ & - & $18(100.0) * * *$ \\
\hline - Mild & $22(46.8)$ & $22(75.9)$ & - \\
\hline - Moderate & $6(12.8)$ & $6(20.7)$ & - \\
\hline - Severe & $1(2.1)$ & $1(3.4)$ & - \\
\hline Erythrocyte sedimentation rate, $\mathrm{mm} / \mathrm{h}^{\mathrm{e}}$ & $5(3-8)$ & $5(4-12)$ & $3.5(2-6) *$ \\
\hline C-reactive protein level, $\mathrm{mg} / \mathrm{l}^{\mathrm{f}}$ & $1(1-1)$ & $1(1-2)$ & $1(1-1)$ \\
\hline
\end{tabular}

$* P<0.050$

$* * P<0.010, * * * P<0.005$

${ }^{a}$ Except where otherwise indicated, values are median (interquartile range)

${ }^{\mathrm{b}}$ Measured on a $0-100 \mathrm{~mm}$ visual analogue scale $(0=$ best, $100=$ worst $)$

${ }^{\mathrm{c}}$ Units; $0=$ best, $3=$ worst

${ }^{\mathrm{d}}$ Overall disease activity of the patient judged by physician, Likert scale

e Normal $<15 \mathrm{~mm} / \mathrm{h}$

${ }^{\mathrm{f}}$ Normal $<0.6 \mathrm{mg} / \mathrm{l}$ 


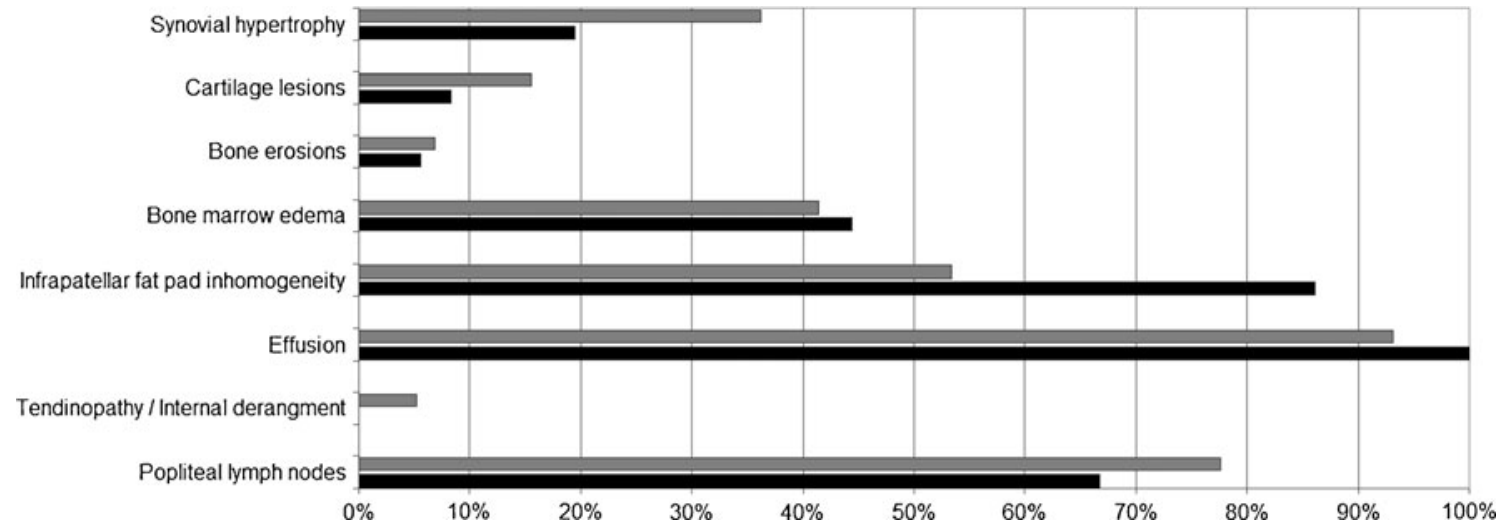

Fig. 1 Proportion of children with JIA showing specific radiological abnormalities. Gray columns for group 1 (active disease) and black columns for group 2 (inactive disease)

Table 2 MRI features in different stages of disease activity in 47 children with juvenile idiopathic arthritis (JIA) ${ }^{\mathrm{a}}$
$* P<0.010, * * P<0.005$

${ }^{a}$ Values are number of knees ( $\%$ of all)

\begin{tabular}{|c|c|c|}
\hline Features & Group 1 (active JIA) 58 knees & Group 2 (inactive JIA) 36 knees \\
\hline Synovial hypertrophy & $21(36.2)$ & $7(19.4)$ \\
\hline Cartilage lesions & $9(15.5)$ & $3(8.3)$ \\
\hline Bone erosions & $4(6.9)$ & $2(5.6)$ \\
\hline Bone marrow changes & $24(41.4)$ & $16(44.4)$ \\
\hline \multicolumn{3}{|l|}{ Infrapatellar fat pad heterogeneity } \\
\hline - Absent & $27(46.6)$ & $5(13.9) *$ \\
\hline - Minimal & $25(43.1)$ & $27(75.0)$ \\
\hline - Marked & $6(10.3)$ & $4(11.1)$ \\
\hline Water & $25(80.6)$ & $16(51.6)$ \\
\hline Scar tissue & $9(29.0)$ & $18(58.1) * *$ \\
\hline Effusion & $54(93.1)$ & $36(100.0)$ \\
\hline \multicolumn{3}{|l|}{ Suprapatellar bursa } \\
\hline - Absent & $16(27.6)$ & $10(27.8)$ \\
\hline - Minimal & $33(56.9)$ & $21(58.3)$ \\
\hline - Marked & $9(15.5)$ & $5(13.9)$ \\
\hline \multicolumn{3}{|l|}{ Lateral recesses } \\
\hline - Absent & $29(50.0)$ & $28(77.8)$ \\
\hline - Minimal & $21(36.2)$ & $8(22.2)$ \\
\hline - Marked & $8(13.8)$ & - \\
\hline \multicolumn{3}{|l|}{ Popliteal cyst } \\
\hline - Absent & $43(74.1)$ & $33(91.7)$ \\
\hline - Minimal & $14(24.1)$ & $3(8.3)$ \\
\hline - Marked & $1(1.7)$ & - \\
\hline \multicolumn{3}{|l|}{ Central recesses } \\
\hline - Absent & $24(41.4)$ & $9(25.0)$ \\
\hline - Minimal & $29(50.0)$ & $22(61.1)$ \\
\hline - Marked & $5(8.6)$ & $5(13.9)$ \\
\hline Tendinopathy/internal derangement & $3(5.2)$ & - \\
\hline \multicolumn{3}{|l|}{ Popliteal lymph nodes } \\
\hline-0 & $13(22.4)$ & $11(30.6)$ \\
\hline-1 & $16(27.6)$ & $9(25.0)$ \\
\hline$-\geq 2$ & $29(50.0)$ & $16(44.4)$ \\
\hline
\end{tabular}


Fig. 2 Sagittal and axial T2weighted fat-saturated MR images (TR/TE, 4,000-6,000/30, TR/TE, 2,900-4,300/50 respectively) obtained in a 16-year-old male (group 1, active disease) show synovial hypertrophy (black arrows) and marked effusion $\left(^{*}\right)$ in the suprapatellar recesses, and synovial hypertrophy (white arrow) adjacent to the medial posterior condyle

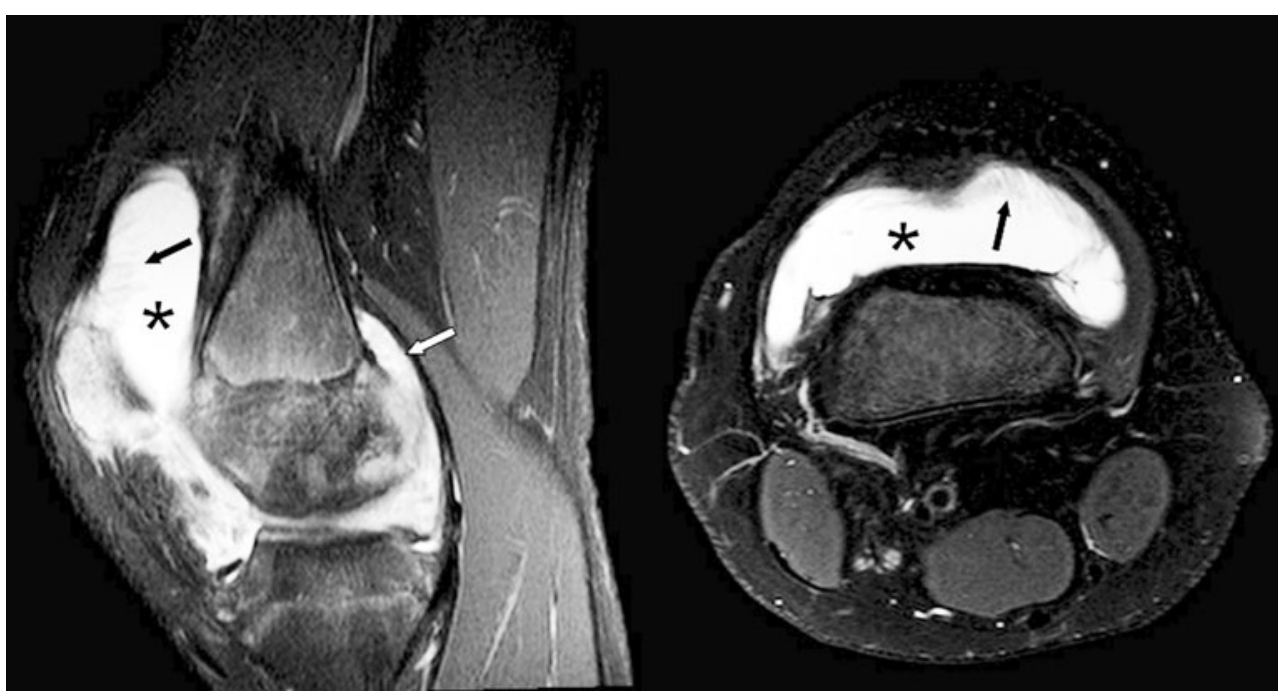

children had a median disease duration of 5.2 years (IQR 1.3-10.7) and median duration of inactive disease of 7.7 months (IQR 5.5-16.5). These data were comparable with those from patients with inactive disease and without signs of synovial hypertrophy (Mann-Whitney $U$ test, $P=$ 0.512 and 0.640 respectively).

Heterogeneity of the infrapatellar fat pad (Fig. 3) was more prevalent in children with clinically inactive disease (86.1\%; groups 1 vs 2 , chi-square test $P=0.008$ ) and correlated well with disease duration. It appeared that fat pad heterogeneity was not related to infiltration of water as a marker of synovitis, but was directly associated with the presence of scar tissue, which also supports the relation with the duration of disease.

Joint effusions were seen in both groups and were predominantly located in the suprapatellar recesses (Fig. 2) and central recesses. No relevant differences were found in groups regarding the presence of cartilage lesions, bone erosions (Fig. 4), bone marrow changes (Fig. 5), joint effusion, tendinopathy/internal derangement of ligaments, or the number of popliteal lymph nodes. Bone erosions were present in six knees on MRI of five children. Corresponding radiographs showed erosions in four knees, whereas two bone erosions seen on MRI were not identified on radiographs (one in both groups).

\section{Reproducibility}

To evaluate reproducibility of the readings, all MR images were reviewed. The overall agreement (Cohen kappa coefficient) on the presence of abnormal MRI features ranged between 0.49 and 0.96 , as shown in Table 3 . Scores of tendinopathy/internal derangement were least consistent.

\section{Discussion}

An MRI protocol was successfully developed for optimizing the feasibility of MRI in children by using an open-bore MRI system and omitting intravenous contrast-medium injection. We could, thereby, take advantage of the benefits of MRI in the evaluation of disease activity in unsedated children with JIA. Our data show that this approach is promising, as all examinations of both knees were successfully completed and no movement artifacts were seen.

We hypothesized that the use of an intravenous contrast agent might reduce patient comfort and feasibility of MRI. Therefore, no intravenous contrast injection was used. However, studies have shown that contrast enhancement facilitates differentiation between joint effusion and synovial hypertrophy through better visualization of hypervascularity of the synovium, reflecting ongoing inflammation $[11,14$, $15,19]$. Therefore, overall presence of synovial hypertrophy may have been underestimated in both groups (active and inactive JIA). Nevertheless, our findings show that even without contrast enhancement, differences in presence of synovial hypertrophy can be detected at various stages of disease activity and substantial reproducibility can be achieved $(\kappa=0.76)$.

So far, the presence of scar tissue in the infrapatellar fat pad has been described in Hoffa disease, after arthroscopy and after surgery [20]. To our knowledge, infrapatellar fat pad scarring has not been previously described as an MRI feature of JIA. In our study, presence of scar tissue in the infrapatellar fat differed significantly between the study groups and correlated with disease duration, suggesting that chronic synovitis eventually results in irreversible soft tissue damage in JIA.

MRI appears more sensitive than clinical examination to detect active joint inflammation [21, 22]. In our cohort, 
Fig. 3 Infrapatellar fat pad heterogeneity caused by water infiltration. a Sagittal T1-weighted and T2-weighted images (TR/TE 450-650/10, TR/TE 4,000-6,000/30, respectively) obtained in a 9-year-old girl (group 1, active disease) show areas of decreased signal intensity on T1-weighted images and increased signal intensity on T2-weighted fat-saturated images (arrows). b Sagittal T1-weighted and T2-weighted images (TR/TE 450-650/10, TR/TE 4,000-6,000/30, respectively) obtained in a 15-year-old male (group 2, inactive disease) show areas of infrapatellar fat pad heterogeneity caused by scar tissue, depicted by decreased signal intensity on both T1-weighted and T2-weighted images (arrows)
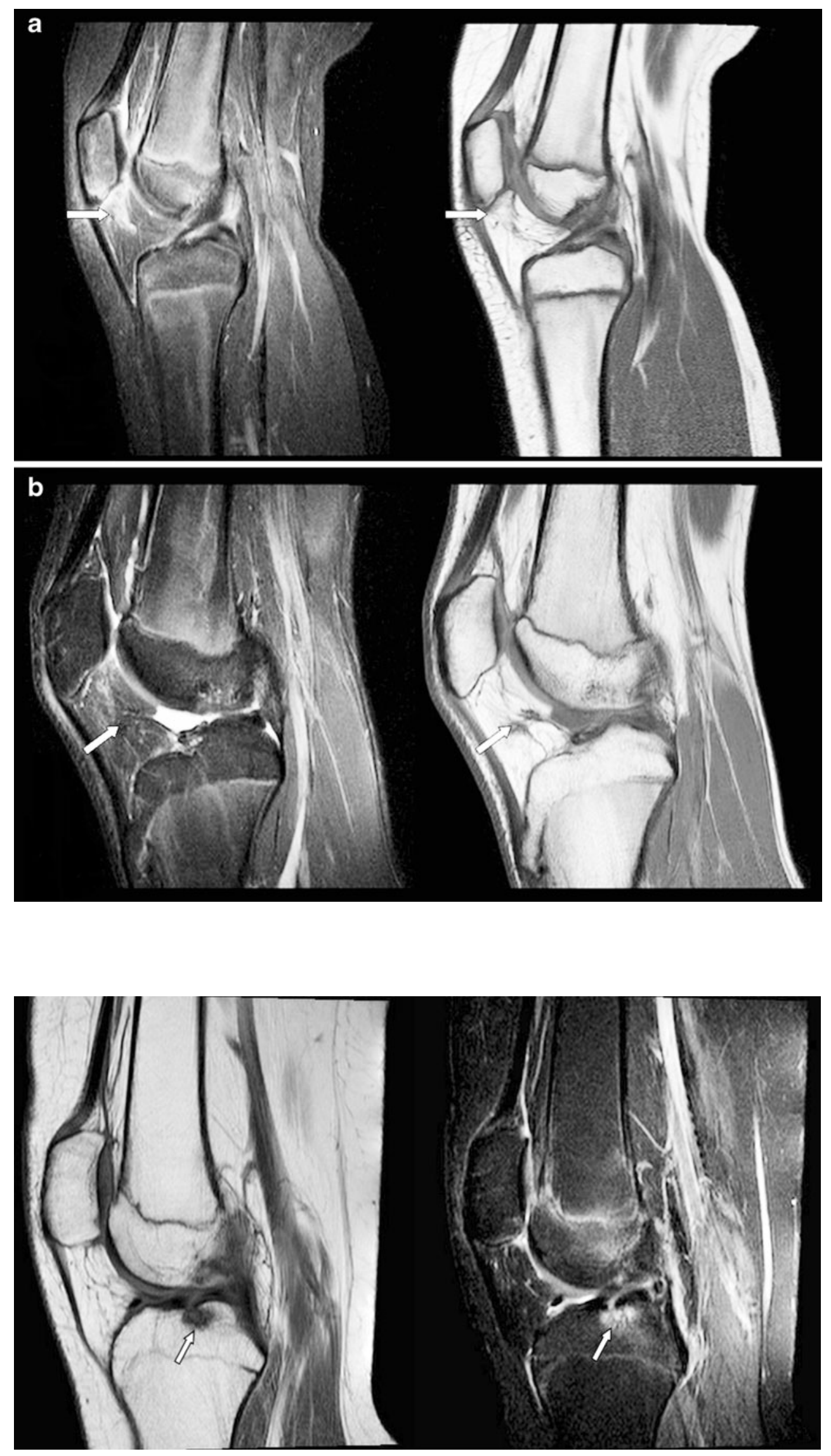

Fig. 4 Sagittal T1-weighted and fat-saturated T2-weighted MR images (TR/TE 450-650/10, TR/ TE 4,000-6,000/30 respectively) obtained in a 15-year-old female (group 1, active disease) show a bone erosion (cortical disruption with accompanying high signal bone marrow changes) in the lateral tibia plateau (white arrow) 
Fig. 5 Bone marrow changes suggestive of bone marrow edema in patients without a history of recent trauma. a Coronal T2-weighted fatsaturated image (TR/TE 2,700$4,500 / 50$ ) obtained in a 14-yearold girl (group 1, active disease) shows an area of increased signal intensity suggestive of bone marrow edema (white arrow) in the lateral tibia plateau. b Coronal T2-weighted fat-saturated image (TR/TE $2,700-4,500 / 50$ ) obtained in a 9-year-old girl (group 1, active disease) shows an area of increased signal intensity suggestive of bone marrow edema (white arrow) in the medial femur condylar
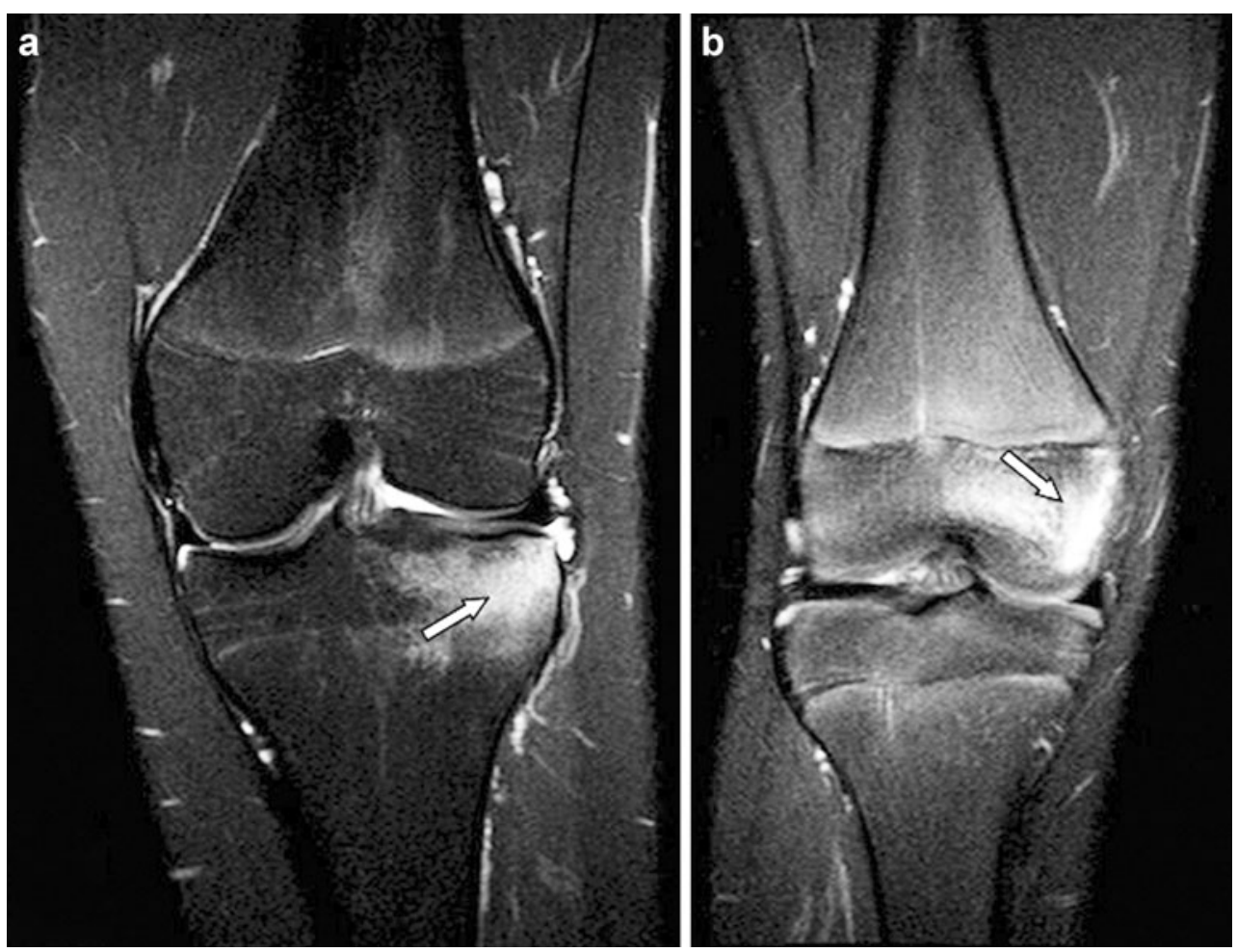

signs of synovial hypertrophy were seen in almost $20 \%$ of the knees of children with clinically inactive disease for at least 6 months (group 2; Table 2) suggesting residual disease activity in $33 \%$ of these children. These results support the use of MRI for accurate evaluation of disease status in JIA. When clinical measures have normalized, MRI may well enable tailoring the therapeutic strategy. The clinical validity and impact of MRI should be formally addressed in prospective outcome studies to compare MRI evaluation directly with clinical scores with respect to decision-making and changes in therapy.

Table 3 Reproducibility of the scoring method

\begin{tabular}{|c|c|}
\hline Feature & Cohen kappa \\
\hline Synovial hypertrophy & 0.76 \\
\hline Cartilage lesions & 0.73 \\
\hline Bone erosions & 0.92 \\
\hline Bone marrow changes & 0.80 \\
\hline Infrapatellar fat pad heterogeneity ${ }^{a}$ & 0.75 \\
\hline - Water & 0.96 \\
\hline - Scar tissue & 0.83 \\
\hline Effusion & 0.85 \\
\hline Tendinopathy/internal derangement & 0.49 \\
\hline Popliteal lymph nodes ${ }^{\mathrm{a}}$ & 0.85 \\
\hline
\end{tabular}

${ }^{a}$ Weighted Cohen kappa
Our study has indicated that creating an MRI scoring system for knee joint pathologies in JIA is possible, with moderate to good reproducibility. However, there were difficulties in the assessment of tendinopathy/internal derangement as suggested by the moderate reliability $(\kappa=0.49)$. Therefore, this feature will be excluded from the score in the future. The scoring system used was intentionally broadly inclusive to capture a wide range of MRI features, therefore refinement of the score is needed and should be addressed in future studies.

Limitations of our study should be considered. The study cohort was relatively small. Moreover, the lack of MR images of age-matched healthy controls is a potential weakness of the study. Growing joints change anatomically, making it difficult to establish whether differences in the appearance of the knee joint are pathological or part of a normal developmental process. There is a high prevalence of bony depressions, signal changes suggestive of bone marrow edema and the presence of joint effusion in the wrists of healthy children. Therefore, such findings should be interpreted with care [4]. On the other hand, it is unlikely that fat pad heterogeneity or synovial hypertrophy will be found in normal unaffected knees. A further limitation is that the presence of synovial hypertrophy could be underestimated because gadolinium enhancement was not applied in the current cohort $[11,14,15,19]$. In an ongoing study, the presence and extent of contrast-enhanced synovial hypertrophy is being addressed. 


\section{Conclusion}

We demonstrated that the use of bilateral non-contrastenhanced open-bore MRI in the assessment of disease activity in JIA is feasible in unsedated children as young as 5 years of age. The presence of synovial hypertrophy and the appearance and presence of soft tissue changes in the infrapatellar fat pad differ in children with JIA between those with active arthritis and those with clinically inactive disease. MRI showed synovial hypertrophy suggestive of inflammation in 33\% of the children with clinically inactive JIA, the clinical implication of which needs further evaluation in prospective studies.

Open Access This article is distributed under the terms of the Creative Commons Attribution Noncommercial License, which permits any noncommercial use, distribution, and reproduction in any medium, provided the original author(s) and source are credited.

\section{References}

1. Ravelli A, Martini A (2007) Juvenile idiopathic arthritis. Lancet 369:767-778

2. Østergaard M, Peterfy C, Conaghan P et al (2003) OMERACT rheumatoid arthritis magnetic resonance imaging studies. Core set of MRI acquisitions, joint pathology definitions, and the OMERACT RA-MRI scoring system. J Rheumatol 30:1385-1386

3. Miller E, Uleryk E, Doria AS (2009) Evidence-based outcomes of studies addressing diagnostic accuracy of MRI of juvenile idiopathic arthritis. AJR 192:1209-1218

4. Müller LS, Avenarius D, Damasio B et al (2011) The paediatric wrist revisited: redefining MR findings in healthy children. Ann Rheum Dis 70:605-610

5. Østergaard M, Conaghan PG, O'Connor P et al (2009) Reducing invasiveness, duration, and cost of magnetic resonance imaging in rheumatoid arthritis by omitting intravenous contrast injection does it change the assessment of inflammatory and destructive joint changes by the OMERACT RAMRIS? J Rheumatol 36:18061810

6. Marshall SP, Smith MS, Weinberger E (1995) Perceived anxiety of pediatric patients to magnetic resonance. Clin Pediatr (Phila) 34:59-60

7. Petty RE, Southwood TR, Manners P et al (2004) International league of associations for rheumatology classification of juvenile idiopathic arthritis: second revision, Edmonton, 2001. J Rheumatol 31:390-392

8. Wallace CA, Ruperto N, Giannini E (2004) Preliminary criteria for clinical remission for select categories of juvenile idiopathic arthritis. J Rheumatol 31:2290-2294

9. Singh G, Athreya BH, Fries JF et al (1994) Measurement of health status in children with juvenile rheumatoid arthritis. Arthritis Rheum 37:1761-1769

10. Wulffraat N, van der Net JJ, Ruperto N et al (2001) The Dutch version of the Childhood Health Assessment Questionnaire (CHAQ) and the Child Health Questionnaire (CHQ). Clin Exp Rheumatol 19:S111-S115

11. Gylys-Morin VM, Graham TB, Blebea JS et al (2001) Knee in early juvenile rheumatoid arthritis: MR imaging findings. Radiology 220:696-706

12. Jacobson JA, Lenchik L, Ruhoy MK et al (1997) MR imaging of the infrapatellar fat pad of Hoffa. Radiographics 17:675-691

13. Cakmakci H, Kovanlikaya A, Unsal E (2001) Short-term followup of the juvenile rheumatoid knee with fat-saturated 3D MRI. Pediatr Radiol 31:189-195

14. El-Miedany YM, Housny IH, Mansour HM et al (2001) Ultrasound versus MRI in the evaluation of juvenile idiopathic arthritis of the knee. Joint Bone Spine 68:222-230

15. Johnson K, Wittkop B, Haigh F et al (2002) The early magnetic resonance imaging features of the knee in juvenile idiopathic arthritis. Clin Radiol 57:466-471

16. Huh YM, Kim S, Suh JS et al (2005) The role of popliteal lymph nodes in differentiating rheumatoid arthritis from osteoarthritis by using CE 3D FSPGR MR imaging: relationship of the inflamed synovial volume. Korean J Radiol 6:117-124

17. van Rossum MA, Zwinderman AH, Boers M et al (2003) Radiologic features in juvenile idiopathic arthritis: a first step in the development of a standardized assessment method. Arthritis Rheum 48:507-515

18. Cohen J (1960) A coefficient of agreement for nominal scales. Educ Psychol Meas 20:307-310

19. Uhl M, Krauss M, Kern S et al (2001) The knee joint in early juvenile idiopathic arthritis. An ROC study for evaluating the diagnostic accuracy of contrast-enhanced MR imaging. Acta Radiol 42:6-9

20. Saddik D, McNally EG, Richardson M (2004) MRI of Hoffa's fat pad. Skeletal Radiol 33:433-444

21. Gardner-Medwin JM, Killeen OG, Ryder CA et al (2006) Magnetic resonance imaging identifies features in clinically unaffected knees predicting extension of arthritis in children with monoarthritis. J Rheumatol 33:2337-2343

22. McQueen FM, Stewart N, Crabbe J et al (1999) Magnetic resonance imaging of the wrist in early rheumatoid arthritis reveals progression of erosions despite clinical improvement. Ann Rheum Dis 58:156-163 